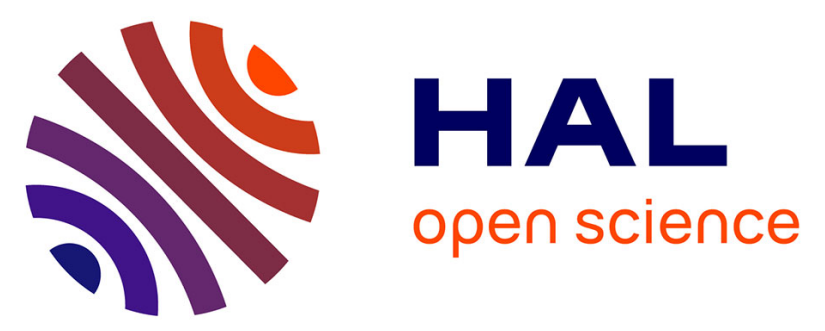

\title{
Double $[3+2]$-dimerisation cascade synthesis of bis(triazolyl)bisphosphanes, a new scaffold for bidentate bisphosphanes
}

Coralie Laborde, Muh-Mei Wei, Arie van Der Lee, Éric Deydier, Jean-Claude Daran, Jean-Noël Null Volle, Rinaldo Poli, Jean-Luc Pirat, Eric Manoury, David Virieux

\section{To cite this version:}

Coralie Laborde, Muh-Mei Wei, Arie van Der Lee, Éric Deydier, Jean-Claude Daran, et al.. Double $[3+2]$-dimerisation cascade synthesis of bis(triazolyl)bisphosphanes, a new scaffold for bidentate bisphosphanes. Dalton Transactions, 2015, 44, pp.12539-12545. 10.1039/C5DT02197A . hal-01203779

\section{HAL Id: hal-01203779 \\ https://hal.science/hal-01203779}

Submitted on 1 Mar 2021

HAL is a multi-disciplinary open access archive for the deposit and dissemination of scientific research documents, whether they are published or not. The documents may come from teaching and research institutions in France or abroad, or from public or private research centers.
L'archive ouverte pluridisciplinaire $\mathbf{H A L}$, est destinée au dépôt et à la diffusion de documents scientifiques de niveau recherche, publiés ou non, émanant des établissements d'enseignement et de recherche français ou étrangers, des laboratoires publics ou privés. 


\section{ARTICLE}

Cite this: DOI: $10.1039 / \times 0 \times x 00000 x$

\section{Double [3+2]-dimerisation cascade synthesis of bis(triazolyl)bisphosphanes, a new scaffold for bidentate bisphosphanes}

Received 00th January 2015, Accepted 00th Marcch 2015

DOI: $10.1039 / \times 0 \times x 00000 x$

www.rsc.org/
Coralie Laborde ${ }^{a}$, Muh-Mei Wei, ${ }^{b}$ Arie van der Lee, ${ }^{c}$ Eric Deydier, ${ }^{b}$ Jean-Claude Daran, ${ }^{b}$ Jean-Noël Volle ${ }^{a}$, Rinaldo Poli, ${ }^{b, d}$ Jean-Luc Pirat, ${ }^{a}$ Eric Manoury, ${ }^{b, *}$ and David Virieux ${ }^{a, *}$

A highly convergent synthesis of bis(triazolylphosphane oxides) was developed by a tandem coppermediated Huisgen reaction - oxidative coupling. The phosphane oxides were reduced by trichlorosilane and the coordination of the resulting bisphosphanes was studied with various transition metals.

\section{Introduction}

The development of innovative, efficient, cost-effective and environmental-friendly processes is essential for the manufacture of new chemical entities. ${ }^{1}$ For this purpose, the processes involving the use of catalysis have clear advantages over those requiring stoichiometric amounts of reagents. ${ }^{2}$ That is why catalysis has become one of the most practical methodologies to build organic molecules, including in enantioselective ways. Most impressive developments have led over the past two decades to numerous products of interest by connecting various building blocks using catalysed hydrogenation or coupling reactions (Heck, Kumada, Negishi, Sonogashira, Stille, Suzuki or Buchwald-Hartwig, etc). In this context, phosphane-based catalysts play an outstanding role because significant achievements have been made in achiral and chiral transition metal catalysed reactions or more recently in phosphane nucleophilic organocatalysed organic transformations. ${ }^{3}$

The design of phosphane ligands has played a key role in the evolution of catalysis. ${ }^{4}$ Fine tuning of the catalytic activity by modification of the electronic/steric properties of the ligand systems is far from trivial mainly because of the difficulty and cumbersome procedures for structural modification of the ligand scaffolds. Therefore, in spite of the large number of developed catalytic processes, much remains to be done in this field in order to develop applicable and highly productive catalysts. Bidentate phosphanes are currently used for their intrinsic chelation properties. Numerous bidentate bisphosphanes are C2-symmetric and $\mathrm{DIOP}^{5}$ or $\mathrm{BINAP}^{6}$ are probably the most known archetypical ligands of this category (fig. 1). Phosphanes for which the phosphino group is directly connected to a heterocyclic ring can take advantage of both electronic and steric modulations induced by the presence of an extra heteroelement. ${ }^{7}$ The dihedral angle in biaryl bisphosphanes has been shown to affect the catalytic properties. ${ }^{8}$ The presence of heteroelements in this biaryl backbone, such as for instance oxygen atoms in SEGPHOS or SYNPHOS, induces stereoelectronic repulsions and consequently narrower dihedral angles. ${ }^{9}$ In this context, still very few phosphanes involves a fivemembered ring as structural subunit. Then axially chiral bisphosphanes derived from 1,1'-naphthyl-benzimidazole core (BIMINAP 1 and BIMIONAP 2) were used as ligands. ${ }^{10}$ Another relevant example, the 2,2'-bis(diphenylphosphanyl)-1,1'-bibenzimidazole (BIMIP 3) ligand is an electron-poor ligand derived from fivemembered bis-heteroaromatic diphosphanes which possess a hindered rotation on the $\mathrm{N}-\mathrm{N}$ linkage. ${ }^{11}$ By contrast, bis(thyenyl)bisphosphanes (BITIOP 4) is considered as electron rich bisphosphane. ${ }^{12}$

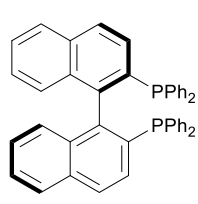

(R)-BINAP

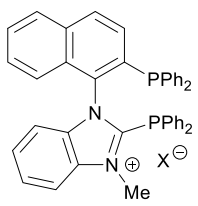

BIMIONAP 2

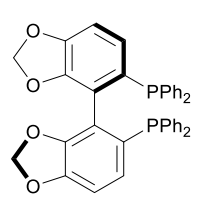

(R)-SEGPHOS

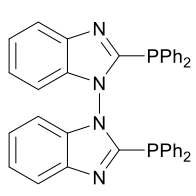

BIMIP 3<smiles>c1ccc(-c2ccc3c(c2-c2ccccc2)OCCO3)cc1</smiles>

(R)-SYNPHOS<smiles>Cc1sc(C)c(-c2c(C)sc(C)c2-c2ccccc2)c1-c1ccccc1</smiles>

BITIOP 4

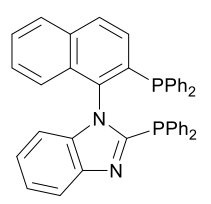

BIMINAP 1

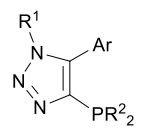

ClickPhos 5
Fig. 1 Representative biaryl bisphosphanes 1-4 and ClickPhos 5.

Zhang and coworkers introduced ClickPhos 5 (i.e. triazolylphosphanes) as potent heterocyclic phosphane ligands. ${ }^{13}$ ClickPhos 5 were synthesized from a two-step procedure using phenyl azide and various aryl acetylenes. They revealed interesting ligand properties, particularly in Suzuki-Miyaura coupling reactions or in amination reactions of aryl halides. ${ }^{14}$ In parallel, extensions of their 
synthesis and luminescence properties were studied by Bräse and coworkers. ${ }^{15}$

\section{Results and discussion}

By contrast, the bis(triazole) backbone is an unprecedented scaffold for the development of new bisphosphane based ligands. We would like to report herein a new class of heterobisphosphanes. Their synthesis is straightforward by a tuneable and convergent methodology which involves the creation of 5 bonds in one chemical step from simple precursors available in large scale using a literature methodology developed with simple substrates. ${ }^{16}$ This reaction was first observed by Sharpless in his early work on click chemistry and was considered at that time as an unwanted side process. ${ }^{17}$

In order to avoid a possible Staudinger reaction, we decided to focus on the combination of diphenylethynylphosphane oxide 6 rather than the free phosphane with alkyl- or arylazides in a tandem coppermediated Huisgen reaction - oxidative coupling, forming bis(triazolylphosphane) oxides 8 (table 1). Diphenylethynylphosphane oxide $\mathbf{6}$ was synthesized in two steps from the reaction of chlorodiphenylphosphane and ethynylmagnesium bromide followed by oxidation of the resulting phosphane with hydrogen peroxide in $78 \%$ overall yield. ${ }^{18}$

The conditions of the tandem [3+2] cyclisation/dimerization were optimized with diphenylethynylphosphane oxide $\mathbf{6}$ and benzylazide 7.1 (table $1, \mathrm{R}=\mathrm{Bn}$ ). Representative assays of this optimization are listed in Table 1. The solvent appeared to have a deep effect on the formation of the desired product. When using a mixture of DMF and water (entries 1-3), only the 1,4-triazole $\mathbf{1 0}$ was formed whatever the carbonate base. THF/water (entries 4-6) gave better results, but the best solvent mixture was acetonitrile/water (entries 7-9). We also explored the effect of the copper catalyst nature. $\mathrm{CuBr}$ alone worked as well as the $\mathrm{Cu}^{0} / \mathrm{CuSO}_{4}$ mixture, even in submolar quantities (entry 8 ). However, the best results were obtained with 1 equivalent of $\mathrm{CuBr}$ (entry 9).

Table 1 Synthesis of bis(triazolylphosphane) dioxides 8.

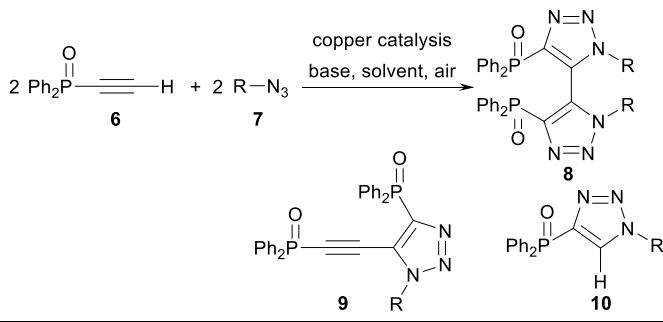

\begin{tabular}{cccccc}
\hline Entry & Base $^{\mathbf{1}}$ & $\begin{array}{c}\text { Eq. } \\
\mathbf{R}^{2} \mathbf{N}^{2}\end{array}$ & Cosolvent $^{\mathbf{3}}$ & $\begin{array}{c}\text { Copper } \\
\text { catalysts }^{4}\end{array}$ & $\begin{array}{c}\text { Product } \\
\text { distribution } \\
\mathbf{8 / 9 / 1 0}[\%]\end{array}$ \\
\hline 1 & $\mathrm{Na}_{2} \mathrm{CO}_{3}$ & 1 & $\mathrm{DMF}$ & $\mathrm{Cu} / \mathrm{CuSO}_{4}$ & $0 / 0 / 100$ \\
2 & $\mathrm{~K}_{2} \mathrm{CO}_{3}$ & 1 & $\mathrm{DMF}$ & $\mathrm{Cu} / \mathrm{CuSO}_{4}$ & $0 / 0 / 100$ \\
3 & $\mathrm{Cs}_{2} \mathrm{CO}_{3}$ & 1 & $\mathrm{DMF}$ & $\mathrm{Cu} / \mathrm{CuSO}_{4}$ & $0 / 0 / 100$ \\
4 & $\mathrm{Cs}_{2} \mathrm{CO}_{3}$ & 1 & $\mathrm{THF}$ & $\mathrm{Cu} / \mathrm{CuSO}_{4}$ & $16 / 74 / 10$ \\
5 & $\mathrm{Cs}_{2} \mathrm{CO}_{3}$ & 1 & $\mathrm{THF}$ & $\mathrm{Cu} / \mathrm{CuSO}_{4}$ & $10 / 71 / 19$ \\
6 & $\mathrm{Cs}_{2} \mathrm{CO}_{3}$ & 5 & $\mathrm{THF}$ & $\mathrm{CuBr}(1 \mathrm{eq})$ & $61 / 35 / 4$ \\
7 & $\mathrm{Na}_{2} \mathrm{CO}_{3}$ & 1 & $\mathrm{ACN}$ & $\mathrm{Cu} / \mathrm{CuSO}$ & $65 / 8 / 30$ \\
8 & $\mathrm{Cs}_{2} \mathrm{CO}_{3}$ & 5 & $\mathrm{ACN}$ & $\mathrm{CuBr}(0.3 \mathrm{eq})$ & $47 / 44 / 8$ \\
9 & $\mathrm{Cs}_{2} \mathrm{CO}_{3}$ & 5 & $\mathrm{ACN}$ & $\mathrm{CuBr}(1 \mathrm{eq})$ & $80 / 11 / 9$
\end{tabular}

1) 2 eq of base were used 2) $R=$ benzyl. 3) 50/50 mixture of the indicated solvent with water. 4) 1 eq / 0.3 eq for the $\mathrm{Cu} / \mathrm{CuSO}_{4}$ mixtures.
Having these optimized conditions in hand, we then changed the nature of the $\mathrm{R}$ group linked to the azide reagents (Table 2). The best yields were obtained for the benzyl and $n$-butyl derivatives $\mathbf{8 . 1}$ and $\mathbf{8 . 2}$ with respectively $49 \%$ and $42 \%$. The reaction tolerated the presence of functional groups such as esters or methylether functions but failed when the reactions were carried out with ketoazides (products 8.8 and 8.9) giving mainly the monotriazole 10 along with some degradation. Compounds 8.1 and 8.3 were crystallized in a form suitable for singlecrystal X-ray diffraction experiments, which confirmed the nature and the positions of the $\mathrm{R}$ group on the triazole ring (Fig. 2).

Table 2 Synthesis of bis(triazolylphosphane) dioxides 8.1-8.7

\begin{tabular}{lccccc}
\hline & $\mathrm{R}$ & Yield $^{1}$ & & $\mathrm{R}$ & Yield \\
\hline $\mathbf{8 . 1}$ & $\mathrm{Bn}$ & 49 & $\mathbf{8 . 6}$ & $\mathrm{CH}_{2} \mathrm{CO}_{2} \mathrm{Me}$ & 20 \\
$\mathbf{8 . 2}$ & $n-\mathrm{Bu}$ & 42 & $\mathbf{8 . 7}$ & $\mathrm{CH}_{2} \mathrm{OBn}$ & 12 \\
$\mathbf{8 . 3}$ & $i$ - $\mathrm{Bu}$ & 23 & $\mathbf{8 . 8}$ & $\mathrm{CH}_{2} \mathrm{C}(\mathrm{O}) \mathrm{Me}$ & - \\
$\mathbf{8 . 4}$ & $c-\mathrm{Hex}$ & 6 & $\mathbf{8 . 9}$ & $\mathrm{CH}_{2} \mathrm{C}(\mathrm{O}) \mathrm{Ph}$ & - \\
$\mathbf{8 . 5}$ & $\mathrm{CH}_{2} \mathrm{CO}_{2} \mathrm{Bn}$ & 29 & & & \\
1) Isolated yield & & & & \\
\hline
\end{tabular}
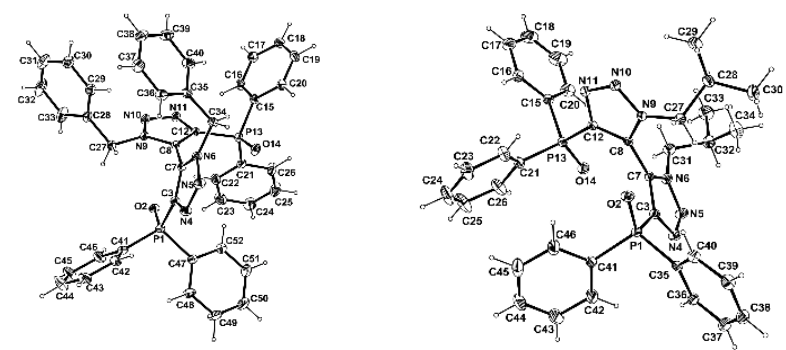

Fig. 2 Views of the molecular geometries of compounds 8.1 and 8.3. The thermal ellipsoids are scaled to a $50 \%$ probability level.

The reduction of the phosphane oxides 8.1-8.3 was accomplished by the classical reaction with trichlorosilane in the presence of triethylamine (Scheme 1). ${ }^{19}$ Under such conditions the resulting bisphosphanes $\mathbf{1 1}$ were isolated in almost quantitative yields.

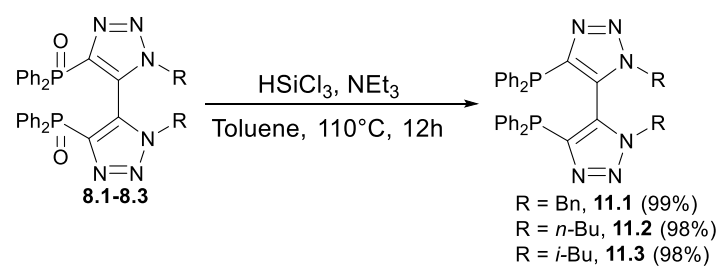

Scheme 1. Reduction of the bis(triazolylphosphane) oxides 8.18.3 to bisphosphanes 11.1-11.3.

The ability of the new triazolylphosphanes to form complexes with different transition metals was also studied (scheme 2). In all cases, ligands 11 bind in the $\kappa^{2}-P, P^{\prime}$ mode without any involvement of the triazole nitrogen atoms. The copper complex 15 was prepared upon reaction of stoichiometric amounts of $\mathbf{1 1 . 1}$ and 1,10-phenanthroline with $\left[\mathrm{Cu}\left(\mathrm{CH}_{3} \mathrm{CN}\right)_{4}\right] \mathrm{BF}_{4}$ in dichloromethane. Similarly, the $\mathrm{Pd}^{\mathrm{II}}$ complexes 12.1-12.3 and 13 were obtained by coordination of ligands 11.1-11.3 to palladium dichloride or bis(acetonitrile) dichloropalladium(II). Platinum complex 14 was also synthesized from 11.1 and bis(acetonitrile)dichloroplatinum(II) and its structure was confirmed by X-ray diffraction (see Supporting Information). Finally, the addition of $\mathbf{1 1 . 1}$ to $[\mathrm{IrCl}(\mathrm{COD})]_{2}$ afforded either complex $[\operatorname{IrCl}(\mathrm{COD})(\mathbf{1 1 . 1})] \mathbf{1 6}$ or the $[\operatorname{Ir}(\mathrm{COD})(\mathbf{1 1 . 1})]^{+}\left[\mathrm{IrCl}_{2}(\mathrm{COD})\right]^{-}$salt 17 when using either a $1: 1$ or a $2: 1 \mathrm{Ir} /$ ligand ratio. The 5-coordinate 
geometry of 16 was confirmed by an X-ray diffraction study (Fig. 3); it is similar to that of other $\left[\mathrm{IrCl}(\mathrm{COD}) \mathrm{L}_{2}\right]$ complexes. ${ }^{20}$ As expected the $\mathrm{Pd}^{\mathrm{II}}$-complex 12.1 showed a narrower dihedral angle of $48.4^{\circ}$ compared to the BINAP-PdCl 2 complex $\left(71.5^{\circ}\right)^{21}$, Synphos $\left(73.6^{\circ}\right)$ or difluorphos $\left(73.0^{\circ}\right)^{9}$ and was a direct consequence of the 5-membered ring backbone rather than the 6-one.

The iridium complex $\mathbf{1 6}$ was tested as a precatalyst in the hydrogenation of N-(1-phenylethylidene)-aniline (see table 3$).{ }^{22}$ Full conversion was achieved at RT under 30 bar hydrogen after $2 \mathrm{~h}$ with only $1 \%$ of complex 16. The in situ made precatalyst (11 $\left./[\operatorname{Ir}(\mathrm{COD}) \mathrm{Cl}]_{2}=1\right)$ was also effective, although slightly less than the complex 16 (table 3, entries 2-5).

Table 3 Hydrogenation of $\mathrm{N}$-(1-phenylethylidene)-aniline with iridium complex 16.

\begin{tabular}{cccc} 
Entry $^{\mathbf{a}}$ & Catalyst & $\begin{array}{c}\text { Time } \\
\text { (h) }\end{array}$ & Yield of amine \\
\hline 1 & $\mathbf{1 6}$ & 0.5 & $57 \%$ \\
2 & $\mathbf{1 6}$ & 2 & $100 \%$ \\
3 & $\begin{array}{c}\mathrm{CH}_{2} \mathrm{Cl}_{2}, \mathrm{RT} \\
4\end{array}$ & 4 & $100 \%$ \\
5 & $\begin{array}{c}\mathbf{1 1} /[\operatorname{Ir}(\mathrm{COD}) \mathrm{Cl}]_{2} \\
(\text { ligand/iridium ratio }=1) \\
\mathbf{1 1} /[\mathrm{Ir}(\mathrm{COD}) \mathrm{Cl}]_{2}(1 / 1) \\
(\text { ligand/iridium ratio }=1)\end{array}$ & 4 & $80 \%$ \\
& & $100 \%$
\end{tabular}

a: $1 \% \mathbf{1 6}, 3 \% \mathrm{I}_{2}$, at $\mathrm{RT}$ in $\mathrm{CH}_{2} \mathrm{Cl}_{2}$.

\section{Conclusions}

In summary, the copper mediated [3+2]-cycloaddition/dimerization of diphenylethynylphosphane oxide with various alkyl azides readily allowed the highly convergent formation of bis(triazolylphosphane) oxides in a one-pot useful process. Reduction into bisphosphanes is very efficient, and coordination of the latter to various transition metals was demonstrated, introducing a new family of $\mathrm{C}_{2}$-symmetric bisphosphanes with a quite rare NCP backbone. Preliminary catalytic studies in imine hydrogenation have shown good catalytic activities with an iridium complex. The determination of configurational stability and the unusual heteroatom backbone could offer numerous perspectives for catalytic applications.

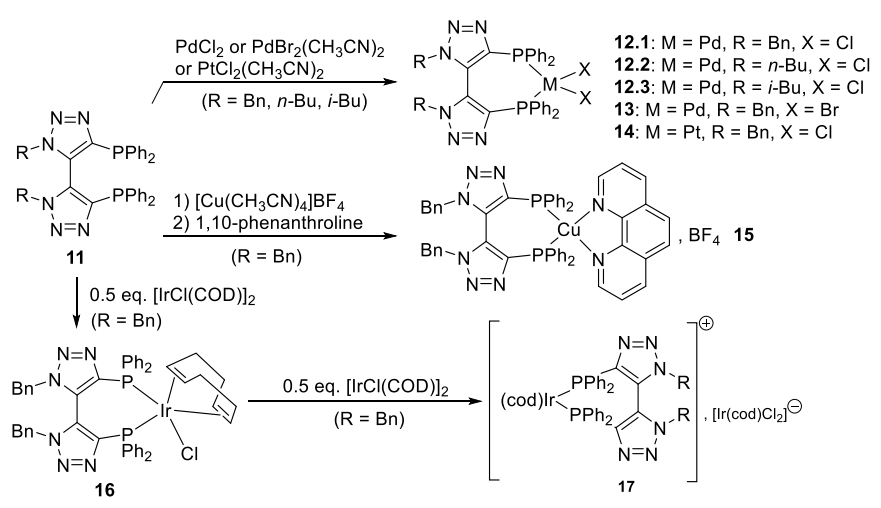

Scheme 2. Synthesis of bis(triazolylphosphane) complexes 1217.

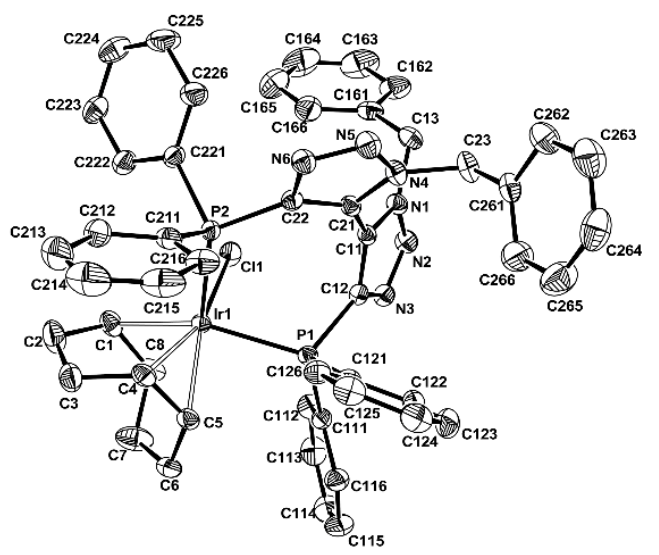

Fig. 3. ORTEP view of the molecular geometry of 16 . The thermal ellipsoids are scaled to a $50 \%$ probability level and the hydrogen atoms are omitted for clarity.

This work was supported by Grants from the Ministère de l'Enseignement Supérieur et de la Recherche, the Centre National de la Recherche Scientifique (CNRS), the "MidiPyrénées" region, and the Institut Universitaire de France.

\section{Experimental}

\section{General}

All reactions were carried out under an argon or nitrogen atmosphere using standard Schlenk techniques otherwise stated. Solvents were carefully dried by conventional or were purified with an MBRAUN Solvent Purification System. ${ }^{1} \mathrm{H},{ }^{13} \mathrm{C}$ and ${ }^{31} \mathrm{P}$ NMR spectra were recorded with a Bruker Avance 500 FT-NMR or a bruker Avance 400 spectrometer. The resonances were calibrated relative to the residual solvent peaks and are reported with positive values downfield from TMS. NMR multiplicities are abbreviated as follows: $\mathrm{s}=$ singlet, $\mathrm{d}=$ doublet, $\mathrm{t}=$ triplet, $\mathrm{q}$ $=$ quartet, $\mathrm{m}=$ multiplet, $\mathrm{br}=$ broad signal. Chemical shifts $(\delta)$ and coupling constants $(\mathrm{J})$ were expressed in $\mathrm{ppm}$ and $\mathrm{Hz}$, respectively. For all characterized compounds, the peak assignments in the ${ }^{1} \mathrm{H}$ and ${ }^{13} \mathrm{C}$ NMR spectra were based on COSY, HSQC and HMBC 2D experiments. HRMS were obtained from dichloromethane solutions with a Xevo G2 Q TOF spectrometer by the electrospray method or with a LC-TOF spectrometer (Micromass).

\section{Synthesis of bistriazolyl phosphane oxides 8.1-8.7}

\section{GENERAL PROCEDURE}

In a round bottom flask, ethynyldiphenylphosphane oxide (250 $\mathrm{mg}, 1.1 \mathrm{mmol})$ was dissolved in acetonitrile $(3 \mathrm{~mL})$. Then the corresponding azide $(3.3 \mathrm{mmol})$ and aqueous cesium carbonate solution at 2 mol. $\mathrm{L}^{-1}(1.65 \mathrm{~mL})$ were added followed by copper bromide (158 mg, $1 \mathrm{mmol})$. The resulting mixture was stirred at room temperature in an open vessel for 12 hours. After complete 
reaction as monitored by TLC, the solvent was removed under reduced pressure and the residue was dissolved in dichloromethane, washed with aqueous ammonia solution $(10 \%$, $3 \times 10 \mathrm{~mL}$ ), brine, then dried over magnesium sulfate and the solvent was evaporated under reduced pressure. The product was isolated as specified below.

\section{4,4'-bis(diphenylphosphinoxy)-1,1'-dibenzyl-5,5'-bis-1,2,3-}

triazole dioxide (8.1). The compound was isolated as a white solid after recrystallization from ethanol; Yield: $49 \%$; ${ }^{1} \mathrm{H}$ NMR $(400,13$ $\left.\mathrm{MHz} \mathrm{CDCl}_{3}\right) \delta 8.13$ - $7.97(\mathrm{~m}, 4 \mathrm{H}), 7.65$ - $7.41(\mathrm{~m}, 10 \mathrm{H}), 7.39-7.08$ $(\mathrm{m}, 12 \mathrm{H}), 6.91-6.77(\mathrm{~m}, 4 \mathrm{H}), 5.51(\mathrm{~d}, J=-14.7 \mathrm{~Hz}, 2 \mathrm{H}), 4.70(\mathrm{~d}, J=$ $-14.7 \mathrm{~Hz}, 2 \mathrm{H}) ;{ }^{13} \mathrm{C}$ NMR $\left(100,61 \mathrm{MHz}, \mathrm{CDCl}_{3}\right) \delta 141.18(\mathrm{~d}, J=130.4$ $\mathrm{Hz}), 132.88$ (s), 132.36 (d, $J=2.8 \mathrm{~Hz}), 132.04$ (d, $J=2.8 \mathrm{~Hz}), 131.65$ (d, $J=10.0 \mathrm{~Hz}$ ), 131.39 (dd, $J=55.3 \mathrm{~Hz}, J=109.07 \mathrm{~Hz}), 131.23$ (d, $J=10.9 \mathrm{~Hz}), 130.85(\mathrm{~d}, J=241.7 \mathrm{~Hz}), 128.92(\mathrm{~s}), 128.83(\mathrm{~s}), 128.62$ (d, $J=12.6 \mathrm{~Hz}), 128.54$ (d, $J=12.8 \mathrm{~Hz}), 128.15$ (s), $53.13(\mathrm{~s}) ;{ }^{31} \mathrm{P}$ NMR (161.97 MHz, $\left.\mathrm{CDCl}_{3}\right) \delta 16.80$ (s); HRMS (EI): $\mathrm{m} / z$ Calcd. for $\mathrm{C}_{42} \mathrm{H}_{34} \mathrm{~N}_{6} \mathrm{O}_{2} \mathrm{P}_{2} \quad[\mathrm{M}+\mathrm{H}]^{+}: 717.22$; Found: 717.2306; mp: 224.5$227.4^{\circ} \mathrm{C}$.

\section{4,4'-bis(diphenylphosphinoxy)-1,1-dibutyl-5,5'-bis-1,2,3-triazole} dioxide (8.2). The compound was isolated as a white solid after purification by a chromatography column on silica gel with as eluent a mixture of dichloromethane-isopropanol (98/2); Yield: $42 \% ;{ }^{1} \mathrm{H}$ NMR (400,13 MHz, $\left.\mathrm{CDCl}_{3}\right) \delta 7.96(\mathrm{dd}, J=7.6 \mathrm{~Hz}, J=12.1 \mathrm{~Hz}, 4 \mathrm{H})$, $7.61-7.35(\mathrm{~m}, 10 \mathrm{H}), 7.33-7.21(\mathrm{~m}, 2 \mathrm{H}), 7.20-7.08(\mathrm{~m}, 4 \mathrm{H}), 4.23$ $4.40(\mathrm{~m}, 2 \mathrm{H}), 4.13-4.01(\mathrm{~m}, 2 \mathrm{H}), 1.92-1.78(\mathrm{~m}, 2 \mathrm{H}), 1.77-1.64(\mathrm{~m}$, 2H), $1.22-1.08(\mathrm{~m}, 4 \mathrm{H}), 0.78(\mathrm{t}, \mathrm{J}=7.4 \mathrm{~Hz}, 6 \mathrm{H}) ;{ }^{13} \mathrm{C}$ NMR $(100,61$ $\left.\mathrm{MHz}, \mathrm{CDCl}_{3}\right) \delta 140.82(\mathrm{~d}, J=130,0 \mathrm{~Hz}), 133.27(\mathrm{~d}, J=2.7 \mathrm{~Hz})$, 131.98 (d, $J=2.7 \mathrm{~Hz}), 131.66(\mathrm{~d}, J=10,0 \mathrm{~Hz}), 131.57$ (dd, $J=29 \mathrm{~Hz}$, $J=110.3 \mathrm{~Hz}), 131.27(\mathrm{~d}, J=10.9 \mathrm{~Hz}), 130.42(\mathrm{~d}, J=21.9 \mathrm{~Hz}), 129,57$ (d, $J=12.7 \mathrm{~Hz}), 129.47$ (d, $J=13,0 \mathrm{~Hz}), 49.40$ (s), 31.07 (s), 19.84 (s), 13.31 (s); ${ }^{31} \mathrm{P}$ NMR (161.97 MHz, CDCl 3 ) $\delta 16,52$ (s); HRMS (EI): $m / z$ Calcd. for $\mathrm{C}_{36} \mathrm{H}_{38} \mathrm{~N}_{6} \mathrm{O}_{2} \mathrm{P}_{2}[\mathrm{M}+\mathrm{H}]^{+}: 649.25$; Found: 649.2607; mp: 172.2-172. ${ }^{\circ} \mathrm{C}$.

\section{4,4'-bis(diphenylphosphinoxy)-1,1'-diisobutyl-5,5'-bis-1,2,3-}

triazole dioxide (8.3). The compound was isolated as a white solid after purification by a chromatography column on silica gel with as eluent a mixture of dichloromethane-isopropanol (98/2); Yield: $23 \%$; ${ }^{1} \mathrm{H}$ NMR $\left(400,13 \mathrm{MHz}, \mathrm{CDCl}_{3}\right) \delta 8.05$ - $7.92(\mathrm{~m}, 4 \mathrm{H}), 7.61$ - $7.44(\mathrm{~m}$, $10 \mathrm{H}), 7.38-7.27(\mathrm{~m}, 2 \mathrm{H}), 7.23-7.14(\mathrm{~m}, 4 \mathrm{H}), 4.20(\mathrm{dd}, J=7.5 \mathrm{~Hz}$, $J=-13.9 \mathrm{~Hz}, 2 \mathrm{H}), 3.88(\mathrm{dd}, J=7.4 \mathrm{~Hz}, J=-13.9 \mathrm{~Hz}, 2 \mathrm{H}), 2.10$ (ht, $J$ $=6.87 \mathrm{~Hz}, J=6.87 \mathrm{~Hz}, 2 \mathrm{H}), 0.81(\mathrm{~d}, J=6.7 \mathrm{~Hz}, 6 \mathrm{H}), 0.73(\mathrm{~d}, J=6.6$ $\mathrm{Hz}, 6 \mathrm{H}) ;{ }^{13} \mathrm{C}$ NMR $\left(100,61 \mathrm{MHz}, \mathrm{CDCl}_{3}\right) \delta 140.87$ (d, $\left.J=131.2 \mathrm{~Hz}\right)$, $133.26(\mathrm{~d}, J=41.9 \mathrm{~Hz}), 132.27(\mathrm{~d}, J=2.7 \mathrm{~Hz}), 132.00(\mathrm{~d}, J=2.8 \mathrm{~Hz})$, $131.71(\mathrm{~d}, J=10.0 \mathrm{~Hz}), 131.22(\mathrm{~d}, J=10.8 \mathrm{~Hz}), 130.67$ (d, $J=22$ $\mathrm{Hz}), 128.58$ (d, $J=12.6 \mathrm{~Hz}), 128.08$ (d, $J=13.0 \mathrm{~Hz}), 56.97$ (s), 28.02 (s), 20.16 (s), 20.03 (s); ${ }^{31} \mathrm{P}$ NMR (161.97 MHz, $\left.\mathrm{CDCl}_{3}\right) \delta 16.5$ (s); HRMS (EI): $m / z$ Calcd. for $\mathrm{C}_{36} \mathrm{H}_{38} \mathrm{~N}_{6} \mathrm{O}_{2} \mathrm{P}_{2}[\mathrm{M}+\mathrm{H}]^{+}: 649.25$; Found: 649.2618; mp: $237.1-238.0{ }^{\circ} \mathrm{C}$.

4,4'-bis(diphenylphosphinoxy)-1,1'-dicyclohexyl-5,5'-bis-1,2,3triazole dioxide (8.4). The compound was isolated as a white solid after sublimation; Yield: 6\%; ${ }^{1} \mathrm{H}$ NMR (400,13 MHz, $\left.\mathrm{CDCl}_{3}\right) \delta 7.95$ 7.89 (qt, 3H), $7.51-7.38$ (m, 11H), $7.27-7.21(\mathrm{~m}, 6 \mathrm{H}), 3.70$ (tt, $J=$
$11.74 \mathrm{~Hz}, 3.70 \mathrm{~Hz}, 2 \mathrm{H}), 2.53-2.56(\mathrm{~m}, 2 \mathrm{H}), 2.11-2.02(\mathrm{~m}, 2 \mathrm{H}), 1.91$ - $1.83(\mathrm{~m}, 2 \mathrm{H}), 1.75-1.66(\mathrm{~m}, 4 \mathrm{H}), 1.56-1.48(\mathrm{~m}, 4 \mathrm{H}), 1.18-1.11$ (m, 2H), $0.99-0.89(\mathrm{~m}, 2 \mathrm{H}), 0,84-0.74(\mathrm{~m}, 2 \mathrm{H}) ;{ }^{13} \mathrm{C}$ NMR $(100,61$ $\left.\mathrm{MHz} \mathrm{CDCl}_{3}\right) \delta 140.47(\mathrm{~d}, J=132.1 \mathrm{~Hz}), 133.13(\mathrm{~d}, J=40.3 \mathrm{~Hz})$, $132.23(\mathrm{~d}, J=2.9 \mathrm{~Hz}), 131.94(\mathrm{~d}, J=2.8 \mathrm{~Hz}), 131.79(\mathrm{~d}, J=10 \mathrm{~Hz})$, $131.56(\mathrm{~d}, J=11 \mathrm{~Hz}), 130.20(\mathrm{~d}, J=21.9 \mathrm{~Hz}), 128.62$ (d, $J=12.6$ $\mathrm{Hz}), 128.40$ (d, $J=13,0 \mathrm{~Hz}), 34.27$ (s), 59.71 (s), 32.35 (s), 25.39 (s), 25.22 (s), 24.89 (s); ${ }^{31} \mathrm{P}$ NMR (161.97 MHz, $\left.\mathrm{CDCl}_{3}\right) \delta$ 17,07 (s); HRMS (EI): $m / z$ Calcd. for $\mathrm{C}_{40} \mathrm{H}_{42} \mathrm{~N}_{6} \mathrm{O}_{2} \mathrm{P}_{2}[\mathrm{M}+\mathrm{H}]^{+}: 701.28$; Found: 701.2908; mp: 282.4-283.0 ${ }^{\circ} \mathrm{C}$.

4,4'-bis(diphenylphosphinoxy)-1,1'-bis(benzyloxyacetyl)-5,5'-bis1,2,3-triazole dioxide (8.5). The compound was isolated as a white solid after purification by a chromatography column on silica gel with as eluent a mixture of dichloromethane-isopropanol (98/2); Yield: $28 \%$; ${ }^{1} \mathrm{H}$ NMR $\left(400,13 \mathrm{MHz}, \mathrm{CDCl}_{3}\right) \delta 7.97$ - $7.89(\mathrm{~m}, 4 \mathrm{H}), 7,59$ $7,52(\mathrm{~m}, 2 \mathrm{H}), 7.51-7.39(\mathrm{~m}, 8 \mathrm{H}), 7.34-7.28(\mathrm{~m}, 8 \mathrm{H}), 7.25-7.19(\mathrm{~m}$, 4H), $7.17-7.09$ (m, 4H), $5.40(\mathrm{~d}, J=-17.8 \mathrm{~Hz}, 2 \mathrm{H}), 5.18(\mathrm{~d}, J=-17.8$ $\mathrm{Hz}, 2 \mathrm{H}), 5.05(\mathrm{~d}, J=-12.1 \mathrm{~Hz}, 2 \mathrm{H}), 4.99(\mathrm{~d}, J=-12.1 \mathrm{~Hz}, 2 \mathrm{H}) ;{ }^{13} \mathrm{C}$ NMR (100,61 MHz, $\left.\mathrm{CDCl}_{3}\right) \delta 165.89$ (s), 141.10 (d, $\left.J=129.7 \mathrm{~Hz}\right)$, 134.58 (s), 132.54 (d, $J=2.7 \mathrm{~Hz}), 132.3$ (dd, $J=20.8 \mathrm{~Hz}, J=110.9$ $\mathrm{Hz}), 132.19$ (d, $J=2.7 \mathrm{~Hz}), 131.83$ (d, $J=10.2 \mathrm{~Hz}), 131.82$ (d, $J=$ $21.7 \mathrm{~Hz}), 131.21$ (d, $J=10.9 \mathrm{~Hz}), 128,77$ (d, $J=12.6 \mathrm{~Hz}), 128.75$ (s), 128,72 (s), 128.55 (s), 128.5 (d, $J=13.0 \mathrm{~Hz}$ ), 68.04 (s), 50.11 (s); ${ }^{31} \mathrm{P}$ NMR (161.97 MHz, $\mathrm{CDCl}_{3}$ ) $\delta 17,36$ (s); HRMS (EI): $\mathrm{m} / z$ Calcd. for $\mathrm{C}_{46} \mathrm{H}_{38} \mathrm{~N}_{6} \mathrm{O}_{6} \mathrm{P}_{2}[\mathrm{M}+\mathrm{H}]^{+}: 833.23$; Found: 833.2400; mp: 157.4-157.9 ${ }^{\circ} \mathrm{C}$.

4,4'-bis(diphenylphosphinoxy)-1,1'-bis(methoxyacetyl) -5,5'-bis1,2,3-triazole dioxide (8.6). The compound was isolated as a white solid after purification by a chromatography column on silica gel with as eluent a mixture of dichloromethane-isopropanol (98/2); Yield: 20\%; ${ }^{1} \mathrm{H}$ NMR $\left(400,13 \mathrm{MHz}, \mathrm{CDCl}_{3}\right) \delta 7.92$ - 7.84 (m, 4H), 7.50 $7.32(\mathrm{~m}, 10 \mathrm{H}), 7.24-7.18(\mathrm{~m}, 2 \mathrm{H}), 7.09-7.03(\mathrm{~m}, 4 \mathrm{H}), 5.29(\mathrm{~d}, J=-$ $17.8 \mathrm{~Hz}, 2 \mathrm{H}), 5.06(\mathrm{~d}, J=-17.8 \mathrm{~Hz}, 2 \mathrm{H}), 3.58(\mathrm{~s}, 6 \mathrm{H}) ;{ }^{13} \mathrm{C} \mathrm{NMR}$ $\left(100,61 \mathrm{MHz}, \mathrm{CDCl}_{3}\right) \delta 166.45(\mathrm{~s}), 147.07$ (d, $\left.J=129.0 \mathrm{~Hz}\right), 132.57$ $(\mathrm{d}, J=2.78 \mathrm{~Hz}), 132.19(\mathrm{~d}, J=2.8 \mathrm{~Hz}), 131.82(\mathrm{~d}, J=10.9 \mathrm{~Hz}), 131.6$ $(\mathrm{dd}, J=23.4 \mathrm{~Hz}, J=110.81 \mathrm{~Hz}), 131.17(\mathrm{~d}, J=10.9 \mathrm{~Hz}), 130.935$ (s), 128,78 (d, $J=12.8 \mathrm{~Hz}), 128.5$ (d, $J=13,0 \mathrm{~Hz}), 52.99$ (s), 49.89 (s); ${ }^{31} \mathrm{P}$ NMR (161.97 MHz, $\left.\mathrm{CDCl}_{3}\right) \delta 17.22$ (s); HRMS (EI): $\mathrm{m} / z$ Calcd. for $\mathrm{C}_{34} \mathrm{H}_{30} \mathrm{~N}_{6} \mathrm{O}_{6} \mathrm{P}_{2}[\mathrm{M}+\mathrm{H}]^{+}: 681.17$; Found: 681.1790; mp: $211.7-212.1^{\circ} \mathrm{C}$.

\section{4,4'-bis(diphenylphosphinoxy)-1,1'-bis(benzyloxymethyl)-5,5'-} bis-1,2,3-triazole dioxide (8.7). The compound was isolated as a white solid after purification by a chromatography column on silica gel with as eluent a mixture of dichloromethane-isopropanol (98/2); Yield: $12 \%$; ${ }^{1} \mathrm{H}$ NMR $\left(400,13 \mathrm{MHz}, \mathrm{CDCl}_{3}\right) \delta 8.04-7.89(\mathrm{~m}, 4 \mathrm{H}), 7.64$ - $7.40(\mathrm{~m}, 10 \mathrm{H}), 7.37-7.11(\mathrm{~m}, 16 \mathrm{H}), 5.87(\mathrm{~d}, J=-11.2 \mathrm{~Hz}, 2 \mathrm{H}), 5.75$ $(\mathrm{d}, J=-11.2 \mathrm{~Hz}, 2 \mathrm{H}), 4.42(\mathrm{~s}, 4 \mathrm{H}) ;{ }^{13} \mathrm{C} \mathrm{NMR}\left(100,61 \mathrm{MHz}, \mathrm{CDCl}_{3}\right) \delta$ 141.51 (d, $J=129.5 \mathrm{~Hz}), 135.72$ (s), 132.43 (d, $J=2.6 \mathrm{~Hz}), 132.07$ $(\mathrm{d}, J=2.7 \mathrm{~Hz}), 131.84(\mathrm{dd}, J=31.1 \mathrm{~Hz}, J=111 \mathrm{~Hz}), 131.78(\mathrm{~d}, J=$ $10.2 \mathrm{~Hz}), 131.22$ (d, $J=10.9 \mathrm{~Hz}), 130.84(\mathrm{dd}, J=21.3 \mathrm{~Hz}), 128.64$ (d, $J=12.8 \mathrm{~Hz}), 128.50$ (s), 128.42 (d, $J=12.9 \mathrm{~Hz}), 128.36$ (s), 128.26 (s), 76.79 (s), 71.29 (s); ${ }^{31} \mathrm{P}$ NMR (161.97 MHz, $\left.\mathrm{CDCl}_{3}\right) \delta 17,34$ (s); HRMS (EI): $m / z$ Calcd. for $\mathrm{C}_{36} \mathrm{H}_{38} \mathrm{~N}_{6} \mathrm{O}_{2} \mathrm{P}_{2}[\mathrm{M}+\mathrm{H}]^{+}: 777.24$; Found: 777.25183; mp: $154.0-154.8^{\circ} \mathrm{C}$. 
Reduction of bistriazolylphosphane oxides 8 into bisphosphanes 11

\section{GENERAL PROCEDURE}

To a mixture of bistriazole (1eq) and triethylamine (12 eq) in anhydrous toluene $\left(0.15 \mathrm{~mol} . \mathrm{ml}^{-1}\right)$ was added dropwise trichlorosilane (10 eq) at room temperature under a nitrogen atmosphere. The mixture was stirred at reflux for $12 \mathrm{~h}$. After complete reduction, observed by ${ }^{31} \mathrm{P} \mathrm{RMN}$, the reaction mixture was quenched with $2 \mathrm{M} \mathrm{NaOH}$ aq and diluted with dichloromethane and water. The organic layer was separated, washed with water and dried over $\mathrm{Na}_{2} \mathrm{SO}_{4}$. After concentration under vacuum, the diphosphane was obtained.

\section{4,4'-bis(diphenylphosphino)-1,1'-dibenzyl-5,5'-bis-1,2,3-triazole}

(11-.1). The compound was isolated as a white solid after precipitation with diethylether and pentane; Yield: 99\%; ${ }^{1} \mathrm{H}$ NMR $(400,13 \mathrm{MHz}$, $\left.\mathrm{CDCl}_{3}\right) \delta 7.68$ - $7.65(\mathrm{~m}, 4 \mathrm{H}), 7.40-7.16(\mathrm{~m}, 22 \mathrm{H}), 6.84-6.82(\mathrm{~m}$, $4 \mathrm{H}), 4.98(\mathrm{~d}, J=-14.9 \mathrm{~Hz}, 2 \mathrm{H}), 4.55(\mathrm{~d}, J=-14.9 \mathrm{~Hz}, 2 \mathrm{H}) ;{ }^{13} \mathrm{C} \mathrm{NMR}$ $\left(100,61 \mathrm{MHz}, \mathrm{CDCl}_{3}\right) \delta 145.74-145.72$ (m (ABX)), 145.57 - 145.56 $(\mathrm{m}(\mathrm{ABX})), 13.49$ - $135.44(\mathrm{~m}(\mathrm{ABX})), 134.92$ - 134.87 (m (ABX)), $134.48(\mathrm{~d}, J=21.7 \mathrm{~Hz}), 133.38$ (s), 133.30 (d, $J=20.7 \mathrm{~Hz}), 130.56$ (d, $J=44.4 \mathrm{~Hz}), 129.14$ (d, $J=65 \mathrm{~Hz}), 128.93$ (s), 128.67 (s), 128.67 - 128.59(m (ABX)), 128.41-128.32 (m (ABX)), 128.22 (s), 52.49 $52.45(\mathrm{~m}(\mathrm{ABX})) ;{ }^{31} \mathrm{P}$ NMR (161.97 $\left.\mathrm{MHz}, \mathrm{CDCl}_{3}\right) \delta-37.97(\mathrm{~s})$; HRMS (EI): $m / z$ Calcd. for $\mathrm{C}_{42} \mathrm{H}_{34} \mathrm{~N}_{6} \mathrm{P}_{2}[\mathrm{M}+\mathrm{H}]^{+}: 685.23$; Found: 685.2398; mp: $205.4-206.5^{\circ} \mathrm{C}$.

\section{4,4'bis(diphenylphosphino)-1,1'-dibutyl-5,5'-bis-1,2,3-triazole}

(11-.2). The compound was isolated as a white solid after precipitation with diethylether and pentane; Yield: $98 \%$; ${ }^{1} \mathrm{H}$ NMR $(400,13 \mathrm{MHz}$, $\left.\mathrm{CDCl}_{3}\right) \delta 7.48-7.58(\mathrm{~m}, 4 \mathrm{H}), 7.30-7.22(\mathrm{~m}, 6 \mathrm{H}), 7.21-7.06(\mathrm{~m}$, $10 \mathrm{H}), 3.96-3.78(\mathrm{~m}, 4 \mathrm{H}), 1.63-1.50(\mathrm{~m}, 4 \mathrm{H}), 1.08-0.89(\mathrm{~m}, 4 \mathrm{H})$, $0.63(\mathrm{t}, J=7.4 \mathrm{~Hz}, 6 \mathrm{H}) ;{ }^{13} \mathrm{C} \mathrm{NMR}\left(100,61 \mathrm{MHz}, \mathrm{CDCl}_{3}\right) \delta 144.02-$ $145.96(\mathrm{~m}(\mathrm{ABX})), 144.88$ - $144.82 \mathrm{~m}(\mathrm{ABX})), 135.51$ - $135.46(\mathrm{~m}$ $(\mathrm{ABX})), 135.08$ - 135.04 (m (ABX)), 134,37 - 134.14 (m (ABX)), $133.48-133.24(\mathrm{~m}(\mathrm{ABX})), 130.64(\mathrm{~d}, J=44.9 \mathrm{~Hz}), 129.11(\mathrm{~d}, J=$ $53.3 \mathrm{~Hz}), 128.71$ - 128.59 (m (ABX)), 128.44 - 128.32 (m (ABX)), 49.07 ( m (ABX)), 31.72 (m (ABX)), 19.81 (s), 13.30 (s); ${ }^{31} \mathrm{P}$ NMR $\left(161.97 \mathrm{MHz}, \mathrm{CDCl}_{3}\right) \delta-37.75$ (s); HRMS (EI): $\mathrm{m} / z$ Calcd. for $\mathrm{C}_{36} \mathrm{H}_{38} \mathrm{~N}_{6} \mathrm{P}_{2}[\mathrm{M}+\mathrm{H}]^{+}$: 617.26; Found: 617.2715; mp: $154.1-155.0{ }^{\circ} \mathrm{C}$.

4,4'bis(diphenylphosphino)-1,1'-diisobutyl-5,5'-bis-1,2,3-triazole (11-.3). The compound was isolated as a white solid after precipitation with diethylether and pentane; Yield: 98\%; ${ }^{1} \mathrm{H}$ NMR $(400,13 \mathrm{MHz}$, $\left.\mathrm{CDCl}_{3}\right) \delta 7.99-7.49(\mathrm{~m}, 4 \mathrm{H}), 7.28-7.23(\mathrm{~m}, 6 \mathrm{H}), 7.22-7.16(\mathrm{~m}$, 4H), $7.16-7.07(\mathrm{~m}, 6 \mathrm{H}), 3.79(\mathrm{dd}, J=7.6 \mathrm{~Hz}, J=13.6 \mathrm{~Hz}, 2 \mathrm{H}), 3.67$ $(\mathrm{dd}, J=7.6 \mathrm{~Hz}, J=13.6 \mathrm{~Hz}, 2 \mathrm{H}), 1.99-1.75(\mathrm{~m}, 2 \mathrm{H}), 0.61$ (d, $J=6.7$ $\mathrm{Hz}, 6 \mathrm{H}), 0.58(\mathrm{~d}, J=6.7 \mathrm{~Hz}, 6 \mathrm{H}) ;{ }^{13} \mathrm{C} \mathrm{NMR}\left(100,61 \mathrm{MHz}, \mathrm{CDCl}_{3}\right) \delta$ 145.15 - 145.06 (m (ABX)), 144.96 - 144.91 (m (ABX)), 135.71 135.67 (m (ABX)), 135.05 - $135.00(\mathrm{~m}(\mathrm{ABX})), 133.56-133.29(\mathrm{~m}$ $(\mathrm{ABX})), 133.13$ - $133.14(\mathrm{~m}(\mathrm{ABX})), 131.20(\mathrm{~d}, J=1.20 \mathrm{~Hz}), 130.74$ $(\mathrm{d}, J=1.37 \mathrm{~Hz}), 129.06(\mathrm{~d}, J=66.8 \mathrm{~Hz}), 128.68-128.50(\mathrm{~m}(\mathrm{ABX}))$, 128.42 - 128.24 (m (ABX)), 56.45 - 56.6 (m (ABX)), 45.85 (s), 28.68 (s), 19.88 (s); ${ }^{31} \mathrm{P}$ NMR (161.97 MHz, $\left.\mathrm{CDCl}_{3}\right) \delta-38.08$ (s) ; HRMS (EI): $m / z$ Calcd. for $\mathrm{C}_{36} \mathrm{H}_{38} \mathrm{~N}_{6} \mathrm{P}_{2}[\mathrm{M}+\mathrm{H}]^{+}: 617.26$; Found: 617.2714 ; mp: $154.2-154.8^{\circ} \mathrm{C}$.

\section{Synthesis of complexes 12-17}

SYNTHESIS OF THE PALLADIUM COMPLEX (13). In a Schlenk tube under argon, a mixture of compound 11.1 (20 mg, $0.029 \mathrm{mmol}$ ) and bis(acetonitrile)dibromopalladium (10.1 $\mathrm{mg}, 0.029 \mathrm{mmol}$ ) was dissolved in dry chloroform $(2 \mathrm{ml})$. The reaction was carried out at room temperature for 2 hours. The solvent was evaporated and the resulting orange solid was washed by dry pentane. After evaporation of the solvent, $22.1 \mathrm{mg}$ of 12.1 were obtained $(80 \%$ yield). ${ }^{1} \mathrm{H}\left\{{ }^{31} \mathrm{P}\right\} \mathrm{NMR}\left(500 \mathrm{MHz}, \mathrm{CDCl}_{3}\right) \delta(\mathrm{ppm}): 7.98(4 \mathrm{H}, \mathrm{m}$, $\left.\mathrm{PPh}_{2}\right), 7.63$ (4H, m, $\left.\mathrm{PPh}_{2}\right), 7.52\left(2 \mathrm{H}, \mathrm{m}, \mathrm{PPh}_{2}\right), 7.49(2 \mathrm{H}, \mathrm{m}$, $\left.\mathrm{PPh}_{2}\right), 7.46$ (4H, m, $\left.\mathrm{PPh}_{2}\right), 7.36$ (4H, m, $\left.\mathrm{PPh}_{2}\right), 7.32$ (2H, m, $\mathrm{Ph} / \mathrm{Bn}), 7.25$ (4H, m, Ph/Bn), 6.77 (4H, m, Ph/Bn), 4.69 (2H, d, $\left.\mathrm{J}=15.7 \mathrm{~Hz}, \mathrm{NCH}_{2}\right), 4.49\left(2 \mathrm{H}, \mathrm{d}, \mathrm{J}=15.7 \mathrm{~Hz}, \mathrm{NCH}_{2}\right) .{ }^{13} \mathrm{C}\left\{{ }^{1} \mathrm{H}\right\}$ NMR (500 MHz, $\left.\mathrm{CDCl}_{3}\right) \delta(\mathrm{ppm}): 141.7\left(\mathrm{~J}_{\mathrm{CP}}=61.1 \mathrm{~Hz}\right.$, quat, $\mathrm{PCN}), 135.8\left(\mathrm{~J}_{\mathrm{CP}}=6.5 \mathrm{~Hz}, \mathrm{PPh}_{2}\right), 133.9\left(\mathrm{~J}_{\mathrm{CP}}=5.0 \mathrm{~Hz}, \mathrm{PPh}_{2}\right)$, $132.9\left(\mathrm{PPh}_{2}\right), 132.1$ (quat, $\left.\mathrm{Ph} / \mathrm{Bn}\right), 131.2\left(\mathrm{PPh}_{2}\right), 129.2\left(\mathrm{~J}_{\mathrm{CP}}=6.3\right.$ $\left.\mathrm{Hz}, \mathrm{PPh}_{2}\right), 129.2(\mathrm{Ph} / \mathrm{Bn}), 129.0(\mathrm{Ph} / \mathrm{Bn}), 128.2$ (quat, $\mathrm{J}_{\mathrm{CP}}=19.9$ $\left.\mathrm{Hz}, \mathrm{PPh}_{2}\right), 127.7\left(\mathrm{~J}_{\mathrm{CP}}=6.0 \mathrm{~Hz}, \mathrm{PPh}_{2}\right), 127.6$ (quat, PCㄷN)127.1 $(\mathrm{Ph} / \mathrm{Bn}), 125.8$ (quat, $\left.\mathrm{J}_{\mathrm{CP}}=40.8 \mathrm{~Hz}, \mathrm{PPh}_{2}\right), 52.5(\mathrm{NCPh}) .{ }^{31} \mathrm{P}\left\{{ }^{1} \mathrm{H}\right\}$ NMR (500 MHz, $\left.\mathrm{CDCl}_{3}\right) \delta(\mathrm{ppm}): 8.4$. HR/MS (ES+) m/e: 871.0541 (M-Br, 100\%) (calc. M: 871.0311).

SYNTHESIS OF THE PLATINUM COMPLEX (14). In a Schlenk tube, under argon, a mixture of ligand $\mathbf{1 1 . 1}(20 \mathrm{mg}, 0.029 \mathrm{mmol})$ and bis(benzonitrile)dichloroplatinum (13.7 mg, $0.029 \mathrm{mmol}$ ) was dissolved in dry chloroform $(2 \mathrm{ml})$. The reaction was carried out at reflux for 2 hours. The solvent was evaporated and the resulting white solid was washed by dry pentane. After evaporation of the solvent, $19.3 \mathrm{mg}$ of 14.1 were obtained $(70 \%$ yield). A single crystal of 14 suitable for X-ray diffraction analysis was obtained by slow diffusion of pentane in a dichloromethane solution. ${ }^{1} \mathrm{H}\left\{{ }^{31} \mathrm{P}\right\} \mathrm{NMR}\left(500 \mathrm{MHz}, \mathrm{CDCl}_{3}\right) \delta$ (ppm): 7.93 (4H, m, PPh $), 7.63$ (4H, m, , $\left.\mathrm{PPh}_{2}\right), 7.50$ (2H, m, , $\mathrm{PPh}_{2}$ ), 7.48 (2H, m, , $\left.\mathrm{PPh}_{2}\right), 7.45$ (4H, m, $\left.\mathrm{PPh}_{2}\right), 7.34$ (4H, m,), 7.33 (2H, m, Ph/Bn), 7.25 (4H, m, Ph/Bn), 6.77 (4H, m, Ph/Bn), $4.74\left(2 \mathrm{H}, \mathrm{d}, \mathrm{J}=15.3 \mathrm{~Hz}, \mathrm{NCH}_{2}\right), 4.60(2 \mathrm{H}, \mathrm{d}, \mathrm{J}=15.3 \mathrm{~Hz}$, $\left.\mathrm{NCH}_{2}\right) .{ }^{13} \mathrm{C}\left\{{ }^{1} \mathrm{H}\right\} \mathrm{NMR}\left(500 \mathrm{MHz}, \mathrm{CDCl}_{3}\right) \delta(\mathrm{ppm}): 140.4\left(\mathrm{~J}_{\mathrm{CP}}=\right.$ $82 \mathrm{~Hz}$, quat, $\mathrm{PCN}), 135.4\left(\mathrm{~J}_{\mathrm{CP}}=6.5 \mathrm{~Hz}, \mathrm{PPh}_{2}\right), 134.2\left(\mathrm{~J}_{\mathrm{CP}}=4.9\right.$ $\left.\mathrm{Hz}, \mathrm{PPh}_{2}\right), 132.8\left(\mathrm{PPh}_{2}\right), 132.2$ (quat, $\left.\mathrm{Ph} / \mathrm{Bn}\right), 131.4\left(\mathrm{PPh}_{2}\right), 129.2$ $(\mathrm{Ph} / \mathrm{Bn}), 129.0\left(\mathrm{PPh}_{2}\right), 129.0(\mathrm{Ph} / \mathrm{Bn}), 127.7\left(\mathrm{~J}_{\mathrm{CP}}=6.0 \mathrm{~Hz}, \mathrm{PPh}_{2}\right)$, $127.6\left(\mathrm{~J}_{\mathrm{CP}}=6.4 \mathrm{~Hz}\right.$, quat, $\left.\mathrm{PCCN}\right), 127.0(\mathrm{Ph} / \mathrm{Bn}), 125.5$ (quat, $\mathrm{J}_{\mathrm{CP}}=29.8 \mathrm{~Hz}, \mathrm{PPh}_{2}$ ), 125.1 (quat, $\mathrm{J}_{\mathrm{CP}}=41.2 \mathrm{~Hz} \mathrm{PPh}_{2}$ ), 52.6 (NCPh). ${ }^{31} \mathrm{P}\left\{{ }^{1} \mathrm{H}\right\}$ NMR (500 $\left.\mathrm{MHz}, \mathrm{CDCl}_{3}\right) \delta(\mathrm{ppm}):-5.1$. HR/MS (ES+) m/e: 915.1645 (M-Cl, 100\%) (calc. M: 915.2381).

SYNTHESIS OF THE IRIDIUM COMPLEX (16). In a Schlenk tube under argon, a mixture of compound $\mathbf{1 1 . 1}$ (20 mg, $0.029 \mathrm{mmol}$ ) and $[\operatorname{Ir}(\operatorname{cod}) \mathrm{Cl}]_{2}(9.8 \mathrm{mg}, 0.015 \mathrm{mmol})$ was dissolved in dry dichloromethane $(2 \mathrm{ml})$. The reaction was carried out at room temperature for 45 minutes. The solvent was evaporated and the resulting yellow solid was washed by dry pentane. After evaporation of the solvent, $29.3 \mathrm{mg}$ of 16 were obtained (99\% yield). A single crystal of 16 suitable for X-ray diffraction analysis has been obtained by slow diffusion of pentane in a 
dichloromethane solution. ${ }^{1} \mathrm{H}\left\{{ }^{31} \mathrm{P}\right\}$ NMR $\left(500 \mathrm{MHz}, \mathrm{CDCl}_{3}\right) \delta$ (ppm): 7.75 (4H, m, PPh $), 7.57$ (4H, m, $\left.\mathrm{PPh}_{2}\right), 7.44(6 \mathrm{H}, \mathrm{m}$, $\left.\mathrm{PPh}_{2}\right), 7.33\left(2 \mathrm{H}, \mathrm{m}, \mathrm{PPh}_{2}\right), 7.25\left(4 \mathrm{H}, \mathrm{m}, \mathrm{PPh}_{2}\right), 7.17(2 \mathrm{H}, \mathrm{m}$, $\mathrm{Ph} / \mathrm{Bn}), 7.07$ (4H, m, Ph/Bn), 6.77 (4H, m, Ph/Bn), 4.65 (2H, d, $\left.\mathrm{J}=15.3 \mathrm{~Hz}, \mathrm{NCH}_{2}\right), 4.58\left(2 \mathrm{H}, \mathrm{d}, \mathrm{J}=15.3 \mathrm{~Hz}, \mathrm{NCH}_{2}\right), 3.67(2 \mathrm{H}$, m, COD), 3.13 (2H, m, COD), 2.00 (2H, m, COD), 1.74 (2H, m, COD), 1.69 (2H, m, COD), $1.53(2 \mathrm{H}, \mathrm{m}, \mathrm{COD}) .{ }^{13} \mathrm{C}\left\{{ }^{1} \mathrm{H}\right\} \mathrm{NMR}$ $\left(500 \mathrm{MHz} \mathrm{CDCl}_{3}\right) \delta(\mathrm{ppm}): 144.9$ (quat, $\mathrm{J}_{\mathrm{CP}}=49.1 \mathrm{~Hz}, \mathrm{PCN}$ ), $135.3\left(\mathrm{~J}_{\mathrm{CP}}=11.7 \mathrm{~Hz}, \mathrm{PPh}_{2}\right), 133.1\left(\mathrm{~J}_{\mathrm{CP}}=9.4 \mathrm{~Hz}, \mathrm{PPh}_{2}\right), 132.8$ (quat, $\mathrm{Ph} / \mathrm{Bn}$ ), 132.6 (quat, $\mathrm{PPh}_{2}$ ), 132.0 (quat, $\left.\mathrm{PPh}_{2}\right), 130.3$ $\left(\mathrm{PPh}_{2}\right), 129.4\left(\mathrm{PPh}_{2}\right), 128.8(\mathrm{Ph} / \mathrm{Bn}), 128.4(\mathrm{Ph} / \mathrm{Bn}), 128.3$ (quat, $\left.\mathrm{J}_{\mathrm{CP}}=26 \mathrm{~Hz}, \mathrm{PC} \underline{\mathrm{CN}}\right) 128.0\left(\mathrm{PPh}_{2}\right), 127.6\left(\mathrm{PPh}_{2}\right), 127.4(\mathrm{Ph} / \mathrm{Bn})$, $68.7\left(\mathrm{~J}_{\mathrm{CP}}=7.9 \mathrm{~Hz}, \mathrm{COD}\right), 67.0\left(\mathrm{~J}_{\mathrm{CP}}=6.8 \mathrm{~Hz}, \mathrm{COD}\right), 52.5$ (NCPh), 32.9 (COD), 32.1 (COD). ${ }^{31} \mathrm{P}\left\{{ }^{1} \mathrm{H}\right\}$ NMR $(500 \mathrm{MHz}$, $\left.\mathrm{CDCl}_{3}\right) \delta(\mathrm{ppm}):-17.8$. HR/MS (ES+) m/e: 985.2919 (M-Cl, 80\%) (calc. M: 985.2888).

SYNTHESIS OF THE IRIDIUM COMPLEX (17). In a Schlenk tube under argon, a mixture of compound 11.1 (20 mg, $0.029 \mathrm{mmol}$ ) and $[\mathrm{Ir}(\mathrm{COD}) \mathrm{Cl}]_{2}(19.6 \mathrm{mg}, 0.029 \mathrm{mmol})$ was dissolved in dry dichloromethane $(4 \mathrm{ml})$. The mixture was stirred at room temperature for 45 minutes. The solvent was then evaporated and the resulting yellow solid was washed by dry pentane. After evaporation of the solvent, $35.4 \mathrm{mg}$ of 17.1 were obtained $(90 \%$ yield). ${ }^{1} \mathrm{H}\left\{{ }^{31} \mathrm{P}\right\}$ NMR (500 $\left.\mathrm{MHz}, \mathrm{CDCl}_{3}\right) \delta(\mathrm{ppm}): 7.67(4 \mathrm{H}, \mathrm{m}$, $\left.\mathrm{PPh}_{2}\right), 7.50$ (4H, m, $\left.\mathrm{PPh}_{2}\right), 7.50$ (2H, m, PPh 2$), 7.31(2 \mathrm{H}, \mathrm{m}$, $\left.\mathrm{PPh}_{2}\right), 7.30$ (4H, m, $\left.\mathrm{PPh}_{2}\right), 7.24\left(4 \mathrm{H}, \mathrm{m}, \mathrm{PPh}_{2}\right), 6.97(2 \mathrm{H}, \mathrm{m}$, $\mathrm{Ph} / \mathrm{Bn}), 6.91$ (4H, m, Ph/Bn), 6.63 (4H, m, Ph/Bn), 5.26 (2H,m, COD), 5.17 (2H, m, COD), $4.92\left(2 \mathrm{H}, \mathrm{d}, \mathrm{J}=15.6 \mathrm{~Hz}, \mathrm{NCH}_{2}\right)$, 4.26 ( $\left.2 \mathrm{H}, \mathrm{d}, \mathrm{J}=15.6 \mathrm{~Hz}, \mathrm{NCH}_{2}\right), 2.68(2 \mathrm{H}, \mathrm{m}, \mathrm{COD}), 2.48(2 \mathrm{H}$, m, COD), 2.37 (2H, m, COD), 2.15 (4H, m, COD), 2.14 (2H, m, COD), 1.98 (2H, m, COD), 1.76 (2H, m, COD), $1.67(2 \mathrm{H}, \mathrm{m}$, COD), 1.45 (2H, m, COD). ${ }^{13} \mathrm{C}\left\{{ }^{1} \mathrm{H}\right\} \mathrm{NMR}\left(500 \mathrm{MHz}, \mathrm{CDCl}_{3}\right) \delta$ (ppm): 140.5 (quat, $\left.\mathrm{J}_{\mathrm{CP}}=70.2 \mathrm{~Hz}, \mathrm{PCN}\right), 137.4\left(\mathrm{~J}_{\mathrm{CP}}=13.6 \mathrm{~Hz}\right.$, $\mathrm{PPh}_{2}$ ), 133.5 (quat, $\left.\mathrm{Ph} / \mathrm{Bn}\right), 132.6\left(\mathrm{~J}_{\mathrm{CP}}=9.0 \mathrm{~Hz}, \mathrm{PPh}_{2}\right), 132.0$ $\left(\mathrm{PPh}_{2}\right), 130.1\left(\mathrm{PPh}_{2}\right), 128.3(\mathrm{Ph} / \mathrm{Bn}), 128.2\left(\mathrm{~J}_{\mathrm{CP}}=11.2 \mathrm{~Hz}, \mathrm{PPh}_{2}\right)$, $127.7\left(\mathrm{~J}_{\mathrm{CP}}=10.8 \mathrm{~Hz}, \mathrm{PPh}_{2}\right), 127.6(\mathrm{Ph} / \mathrm{Bn}), 127.3(\mathrm{Ph} / \mathrm{Bn}), 127.1$ (quat, $\mathrm{J}_{\mathrm{CP}}=11.0 \mathrm{~Hz}, \mathrm{PCCN}$ ), 125.3 (quat, $\mathrm{J}_{\mathrm{CP}}=53.9 \mathrm{~Hz}, \mathrm{PPh}_{2}$ ), $94.8\left(\mathrm{~J}_{\mathrm{CP}}=13.4 \mathrm{~Hz}, \mathrm{COD}\right), 94.3\left(\mathrm{~J}_{\mathrm{CP}}=16.1 \mathrm{~Hz}, \mathrm{COD}\right), 55.4$ (COD), 53.2 (COD), 51.7 (NCPh), 34.3 (COD), 32.1 (COD), 29.9 (COD), 29.1 (COD). ${ }^{31} \mathrm{P}\left\{{ }^{1} \mathrm{H}\right\} \mathrm{NMR}\left(500 \mathrm{MHz}, \mathrm{CDCl}_{3}\right) \delta$ (ppm): 8.7. HR/MS (ES+) m/e: 985.2916 (M of cation, 100\%) (calc. M: 985.2888).

HYDROGENATION OF N-(1-PHENYLETHYLIDENE)-ANILINE WITH COMPLEX (16). The catalyst $(6.4 \mu \mathrm{mol}$ : $6.5 \mathrm{mg}$ of complex 16 or $4.4 \mathrm{mg}$ of ligand $\mathbf{1 1 b}$ and $2.2 \mathrm{mg}$ of $\left.[\operatorname{Ir}(\mathrm{COD}) \mathrm{Cl}]_{2}\right), \mathrm{N}-(1-$ phenylethylidene)-aniline (0.64 mmol, $125 \mathrm{mg}$ ) and $\mathrm{I}_{2}(19.2$ $\mu \mathrm{mol}, 4.9 \mathrm{mg}$ ) were placed in a vial which was placed into an autoclave. The autoclave was pressurized with $\mathrm{H}_{2}$ (30 bar) and the mixture was then stirred at room temperature. After the indicated time, the autoclave was vented and the recovered mixture was filtered through a short silica gel column before being analysed by GC.

\section{Crystallographic studies}

A single crystal of each compound was mounted under inert perfluoropolyether at the tip of a cryoloop and cooled in the cryostream of either an Oxford-Diffraction XCALIBUR SAPPHIRE-I CCD diffractometer or an Agilent Technologies GEMINI EOS CCD diffractometer. Data were collected using the monochromatic MoKa radiation $(1=0.71073)$. The structures were solved by direct methods (SIR97) ${ }^{23}$ and refined by leastsquares procedures on F2 using SHELXL-97. ${ }^{24}$ All $\mathrm{H}$ atoms attached to carbon were introduced in idealised positions and treated as riding on their parent atoms in the calculations. The drawing of the molecules was realised with ORTEP3. ${ }^{24}$ CCDC reference numbers are 1031965-1031970.

\section{Notes and references}

a Institut Charles Gerhardt / UMR 5253 / AM2N, Ecole Nationale Supérieur de Chimie de Montpellier, 8, Rue de l'Ecole Normale, 34296 Montpellier Cedex 5, France.

${ }^{b}$ CNRS, LCC (Laboratoire de Chimie de Coordination) - Université de Toulouse, UPS, INPT - 205 route de Narbonne, BP 44099, F-31077 Toulouse Cedex 4, France.

${ }^{c}$ Institut Européen des Membranes, CNRS - UMR 5635, Université de Montpellier II - Case Courrier 047 Place E. Bataillon, F-34095 MONTPELLIER Cedex 5, France.

${ }^{d}$ Institut Universitaire de France, 103 bd Saint-Michel, F-75005 Paris, France.

1 A. Behr, A. J. Vorholt, K. A. Ostrowski and T. Seidensticker, Green Chem., 2014, 16, 982; A. C. Jones, J. A. May, R. Sarpong and B. M. Stoltz, Angew. Chem. Int. Ed., 2014, 53, 2556; P. T. Anastas, and M. M. Kirchhoff, Acc. Chem. Res., 2002, 35, 686; R. A. Sheldon, Chem. Ind. (London), 1992, 903.

2 H.-U. Blaser, F. Spindler and M. Studer, Appl. Catal., 2001, A221, 119.

3 For organocatalysis see: C. M. R. Volla, J. Atodiresei and M. Rueping, Chem. Rev., 2014, 114, 2390; S. E. Denmark and G. L. Beutner, Angew. Chem. Int. Ed., 2008, 47, 1560; J. L. Methot and W. R. Roush, Adv. Synth. Catal., 2004, 346, 1035.

4 J. F. Hartwig, Organotransition Metal Chemistry, from Bonding to Catalysis, University Science Books: New York, 2010.

5 T. D. Dang and H. B. Kagan, Chem. Commun., 1971, 481.

6 A. Miyashita, A. Yasuda, H. Takaya, K. Toriumi, T. Ito, T. Souchi and R. Noyori, J. Am. Chem. Soc., 1980, 102, 7935.

7 S. M. Wong, C. M. So and F. Y. Kwong, Synlett, 2012, 1132; Y. Canac and R. Chauvin, Eur. J. Inorg. Chem., 2010, 2325.

8 Z. Freixa and P. W. N. M. van Leeuwen, Dalton Trans, 2003, 1890.

9 T. Saito, T. Yokozawa, T. Ishizaki, T. Moroi, N. Sayo, T. Miura and H. Kumobayashi, Adv. Synth. Catal., 2001, 343, 264; D. E. Kim, C. Choi, I. S. Kim, S. Jeulin, V. Ratovelomanana-Vidal, J.-P. Genêt and N. Jeong, Adv. Synth. Catal., 2007, 349, 1999.

10 N. Debono, Y. Canac, C. Duhayon, R. Chauvin, Eur. J. Inorg. Chem. 2008, 2991; I. Abdellah, N. Debono, Y. Canac, L. Vendier, R. Chauvin, Chem. Asian. J. 2010, 1225. 
11 T. Benincori, E. Brenna, F. Sannicolò, L. Trimarco, P. Antognazza, E. Cesarotti, F. Demartin, T. Pilati, G. Zotti, J. Organomet. Chem., 1997, 529, 445.

12 T. Benincori, E. Cesarotti, O. Piccolo, F. Sannicolo, J. Org. Chem., 2000, 65, 2043.

13 D. Liu, W. Gao, Q. Dai and X. Zhang, Org. Lett., 2005, 7, 4907; Penn State Research Foundation, Patent: WO2006/130842 A1, 2006. Other triazolylphosphanes are also described. For Clickphines see R. J. Detz, S. Arévalo Heras, R. de Gelder, P. W. N. M. van Leeuwen, H. Hiemstra, J. N. H. Reek and J. H. van Maarseveen, Org. lett., 2006, 8, 3227. For ChiraClick ligands see: F. Dolhem, M. J. Johansson, T. Antonsson and N. Kann, J. Comb. Chem., 2007, 9, 477; S. G. A. van Assema, C. G. J. Tazelaar, G. Bas de Jong, J. H. van Maarseveen, M. Schakel, M. Lutz, A. L. Spek, J. C. Slootweg and K. Lammertsma, Organometallics, 2008, 27, 3210.

14 Q. Dai, W. Gao, D. Liu, L. M. Kapes and X. Zhang, J. Org. Chem., 2006, 71, 3928.

15 D. Zink, T. Baumann, M Nieger and S. Bräse, Eur. J. Org. Chem., 2011, 1432; D. M. Zink, T. Grab, T. Baumann, M. Nieger, E. C. Barnes, W. Klopper and S. Bräse, Organometallics, 2011, 30, 1432.

16 Y. Angell and K. Burgess, Angew. Chem. Int. Ed., 2007, 46, 3649 ; J. González, V. M. Pérez, D. O. Jiménez, G. Lopez-Valdez, D. Corona and E. Cuevas-Yañez, Tetrahedron Lett., 2011, 52, 3514.

17 V. V. Rostovtsev, L. G. Green, V. V. Fokin and K. B. Sharpless, Angew. Chem Int. Ed., 2002, 41, 2596.

18 V. Huc, A. Balueva, R. M. Sebastian, A. M. Caminade, and J.P.Majoral, Synthesis, 2000, 26.

19 Q.-Y. Zhao, Z.-L. Yuan and M. Shia, Adv. Synth. Cat., 2011, 353, 637; W. Tang, B. Qu, A. G. Capacci, S. Rodriguez, X. Wei, N. Haddad, B. Narayanan, S. Ma, N. Grinberg, N. K. Yee, D. Krishnamurthy and C. H. Senanayake, Org. Lett., 2010, 12, 176; S. Duprat de Paule, S. Jeulin, V. Ratovelomanana-Vidal, J.-P. Genet, N. Champion and P. Dellis, Eur. J. Org. Chem., 2003, 1931. Y. Uozumi and T. Hayashi, J. Am. Chem. Soc., 1991, 113, 9887.

20 T. Hayashi, M. Tanaka, I. Ogata, T. Kodama, T. Takahashi, Y. Uchida and T. Uchida, Bull. Chem. Soc. Japan, 1983, 56, 1780-1785; T. Shibata, K. Yamashita, H. Ishida and K. Takagi, Org. Lett., 2001, 3 , 1217-1219; M. Brym and C. Jones, Trans. Met. Chem., 2003, 28, 595; R. Malacea, E. Manoury, L. Routaboul, J.-C. Daran, R. Poli, J. P. Dunne, A. C. Withwood, C. Godard, S.B. Duckett, Eur. J. Inorg. Chem. 2006, 1803.

21 A. C. Vérone, M. Felber, O. Blaque, B. Spingler, Polyhedron, 2013, $\mathbf{5 2}, 102$.

22 For recent reviews on metal-catalyzed hydrogenation of imines: (a) K. H. Hopmann, A. Bayer, Coord. Chem. Rev., 2014, 268, 59. (b) N. Fleury-Bregeot, V. de la Fuente, S. Castillon, C. Claver, ChemCatChem, 2010, 2, 1346. (c) A. Fabrello, A. Bachelier, M. Urrutigoity, P. Kalck, Chem. Coord. Rev., 2010, 254, 273.

22 A. Altomare, M. C. Burla, M. Camalli, G. L. Cascarano, C. Giacovazzo, A. Guagliardi, A. G. G. Moliterni, G. Polidori and R. Spagna, J. Appl. Cryst. 1999, 32, 115. SIR97- a program for automatic solution of crystal structures by direct methods.

23 G. M. Sheldrick, Acta Cryst.A 2008, 64, 112.

24 M. N. Burnett and C. K. Johnson, 1995, ORTEPIII, Report ORNL6895. Oak Ridge National Laboratory, Oak Ridge, Tennessee, U.S. L. J. Farrugia, J. Appl. Cryst. 1997, 30, 565. ORTEP-3 for Windows. 


\section{ARTICLE}

\section{Graphical Abstract}

Double [3+2]-dimerisation cascade synthesis of bis(triazolyl)bisphosphanes, an unprecedented scaffold for bide ntate bisphosphanes

C. Laborde, M.-M. Wei, A. van der Lee, E. Deydier, J.-C. Daran, J.-N. Volle, R. Poli, J.-L. Pirat, E. Manoury and D. Virieux

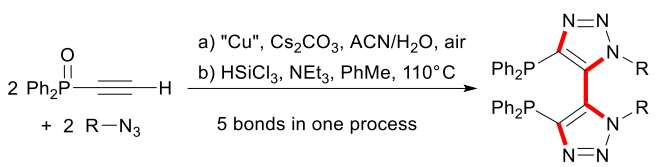

Highly convergent synthesis of bis(triazolylphosphane oxides) by a tandem copper-mediated Huisgen reaction - oxidative coupling. 